\title{
A Cross-Sectional Survey of the Workplace Factors Contributing to Symptoms of Anxiety and Depression Among Nurses and Physicians During the First Wave of COVID-19 Pandemic in Two US Healthcare Systems
}

\section{Igor Burstyn ${ }^{1, *, 0}$ and Karyn Holt ${ }^{2}$}

'Department of Environmental and Occupational Health, Dornsife School of Public Health, Drexel University, Philadelphia, PA 19104, USA; ${ }^{2}$ School of Nursing, the University of Nevada Las Vegas, Las Vegas, NV 89154, USA

*Author to whom correspondence should be addressed. Tel: +1-215-762-2267; e-mail: ib86@drexel.edu

Submitted 9 May 2021; revised 19 August 2021; editorial decision 7 September 2021; revised version accepted 15 September 2021.

\begin{abstract}
Background: Anxiety and depression among physicians and nurses during the COVID-19 pandemic in the USA are not well described and their modifiable causes are poorly understood.

Methods: We conducted a cross-sectional survey of symptoms of anxiety and depression (Hospital Anxiety and Depression Scale) among physicians and nurses in two US healthcare systems in June through September 2020; participation rate was $5-10 \%$. We described features of work as well as their perceptions and associated concerns in relation to the risk of anxiety and depression, while controlling for health history via regression and path analyses.

Results: About a third of 684 nurses and 185 physicians surveyed showed symptoms of anxiety or depression, and the excess of symptoms of mood disorders was particularly prominent in nurses. The belief that one was infected was a dominant correlate of both anxiety and depression. This belief was more associated with history of symptoms of pneumonia than the contact with COVID19 diagnosed patients. Factors found to be associated with reduced anxiety and depression in this working environment were having confidence in the competent use of and access to personal protective equipment, maintaining usual working hours, being surrounded by colleagues who were both sufficient in numbers and not stressed, and the support of immediate family and religious communities. Involvement in aerosol-generating procedures with infected patients was linked with lower depression in nurses but higher among physicians. Likewise, the setting of recent patient encounters affected risk of anxiety and depression differently for physicians and nurses.
\end{abstract}

(C) The Author(s) 2021. Published by Oxford University Press on behalf of the British Occupational Hygiene Society. 


\section{What's Important About This Paper?}

About a third of nurses and physicians surveyed in two US healthcare systems showed symptoms of anxiety or depression. Factors associated with anxiety and depression may help develop mitigation measures. This study underscores the need to help nurses and physicians bear the psychological burden of COVID-19 pandemic and similar events in the future.

Conclusions: Our findings may help develop mitigation measures and underscore the need to help nurses and physicians bear the psychological burden of the COVID-19 pandemic and similar events in the future.

Keywords: anxiety; COVID-19; depression; epidemic; epidemiology; healthcare workers; mood disorders; mental health; nurses; physicians; pandemic

\section{Introduction}

Healthcare workers (HCW) are presumed to be at risk for nosocomial infections with SARS-CoV-2, virus causing COVID-19 disease. There is a robust and widely publicized evidence that HCWs in the USA are at increased risk for COVID-19 disease. According to the US Center for Disease Control, $55 \%$ of HCW with COVID19 reported contact with infected individuals only in a healthcare setting and they were the dominant occupational group among diagnosed cases during the onset of the pandemic (CDC, 2020). The infection rate for SARS$\mathrm{CoV}-2$ in the early days of the pandemic was $7.3 \%$ in one study of the US HCWs while only affecting $0.4 \%$ of others (Barrett et al., 2020), but continued to increase, as the pandemic progressed towards its second wave, among HCWs, with nurses being reported as having the highest infection rates (Rebmann, 2020).

Given that HCWs understand better than most that they are at an elevated risk of any infection during an outbreak of a novel infection, they can be expected to be at risk for psychological distress, because they themselves become infected as was the cases during SARS pandemic (Maunder et al., 2004; Lee et al., 2007). For example, Chong et al. (2004) reported pervasive emotional distress, feelings of extreme vulnerability, uncertainty, and threats to life, among HCWs during the rapid spread of SARS. Soklaridis et al. (2020) argued that review of evidence available as of June 2020 indicates that HCWs in general are among those who are particularly distressed and fearful during pandemics, aggravated by many factors, including concerns about workload, exposure, shortages of personal protective equipment (PPE), and inadequate support. The authors identified lack of consideration of pre-existing medical conditions as one of the weaknesses in available evidence and emphasized that cultural context must be considered, implying the need for local data to inform mitigation measures. Synthesis of relevant literature on COVID19 and earlier similar outbreaks by Preti et al. (2020) reveals elevated anxiety and depression among HCWs, mitigated by a plethora of work-related factors such as support and confidence in PPE, some of these factors presumably modifiable. With the SARS outbreak, new onset mental ill-health was not more common 1 year later in HCW involved in the care of SARS patients than rates in the community (Lancee et al., 2008). This may not be a valid prediction of effects with the COVID-19 pandemic, because it has had longer disruptive effects on the lives of a greater number of HCWs.

Meta-analysis of studies from China and Singapore by Pappa et al. (2020) suggests high levels of anxiety and depression among HCW involved in the care of patients with COVID-19 early in the pandemic, with somewhat higher levels among nurses (26-30\%) compared to physicians $(22-25 \%)$; the risk was higher on average among female physicians and nurses. In one study included in the meta-analysis by Pappa et al. (2020), Lai et al. (2020) reported that among 1,257 HCWs in 34 hospitals in China, during January to February 2020, the symptoms of anxiety and depression (exhibited by more about half of the participants) were elevated on average by $50 \%$ among those who were engaged in direct care of COVID-19 patients; higher known infection rates in a region where HCWs practiced adversely affected mental health. Likewise, in the largest study included in the meta-analysis of 11,118 HCWs in China (Guo et al., 2020), authors reported that among 3,351 frontline HCWs there was on average doubling of "severe" anxiety and depression compared to non-frontline HCWs. Wang et al. (2020) 
observed that poor self-rated health, having a chronic illness, suspected contact with COVID-19 diagnosed person, and specific symptoms of ill-health consistent with COVID-19 during previous 14 days were associated with elevated symptoms of anxiety and depression in a general population sample in China of 1,210 respondents from 194 cities collected in February 2020, suggesting that the same associations may also exist among HCWs. The perception of lack of adequacy of PPE and infection control during the COVID-19 pandemic was associated with increased symptoms of anxiety and depression among 5,988 Canadian HCWs during spring of 2020 (Smith et al., 2020).

In the USA, a nation-wide convenience sample (high in emergency department staff) of 2,040 HCWs during May 2020 noted that having reported symptoms consistent with COVID-19 was associated with anxiety and depression (Firew et al., 2020). It must be noted that almost a third of the participants were suspected of having COVID-19, a far higher rate than expected from a random sample at the time, further limiting work's generalizability to the typical situation of far lower infection rates; pre-existing factors and conditions such as anxiety, depression, and perception of mental health support were not evaluated. First responders, including 98 hospital staff, from the Rocky Mountain region of the USA during spring of 2020 (Wright et al., 2020), exhibited evidence of higher levels of anxiety and depression due to contact with COVID-19 patients and their own reported immunocompromised status. Czeisler et al. (2020) provide evidence of increase in anxiety and depression in the USA in general during April to June 2020 compared to the same period a year before, with a notable excess of having considered suicide among essential workers. The survey highlighted importance of adjusting for factors and conditions such as history of anxiety and depression, including whether it was recently treated. Overall, data on anxiety and depression among HCWs in USA during COVID-19 pandemic are limited, with few indications of whether modifiable causes are seen in other populations are at play.

We aim to identify workplace factors that place physicians and nurses at risk for anxiety and depression during the first wave of the COVID-19 pandemic in samples from two healthcare systems in the USA, accounting for health history, perceived risks, and available support. We are particularly interested in the role that recent personal health and belief about having been infected may be related to anxiety, because this belief can be addressed through workplace policies on testing and can be used to identify persons in need of support.

\section{Materials and methods}

Our project received ethics approval from the Institutional Review Boards of the respective institutions.

\section{Study design and settings}

We designed a cross-sectional survey of all physicians and nurses employed and contracted by the Tower Health in Southeastern Pennsylvania (TH) and the University Medical Center, Las Vegas, Nevada (UMC), and licensed to practice in these states, corresponding to the early phases of the COIVD-19 pandemic in the USA. Physicians and nurses were recruited through Health Systems' employee databases. The complexity and technical nature of the questionnaires made it inappropriate to deliver to a wider range of healthcare employees who were also on the frontlines, but not accessible to these researchers. Participation was both voluntary and confidential, unless the participants chose to enter their name in the survey wishing to be contacted for participation in a follow-up study (yet to be conducted). TH is a regional healthcare provider that offers healthcare and wellness services to a population of 2.5 million people in Philadelphia and Southeastern Pennsylvania. It includes six acute care hospitals and other entities that provide a full range of medical care, wellness programs, and public health services. TH consists of numerous hospitals, including a pediatric hospital, a partnership with Drexel University, in Philadelphia, home healthcare services, and a network of 22 urgent care facilities. The UMC is an academic health care center and the anchor hospital of the Las Vegas Medical District, and the only level one trauma center in Las Vegas, with 564 total hospital beds. It is the eighteenth largest public hospital in the USA, providing both adult and pediatric care over portions of Nevada, California, Arizona, and Utah.

\section{Data collection}

We collected data via an online survey (implemented in Qualtrics hosted by Drexel University). The invitation to enroll in the study was distributed by email, using mailing lists held by TH and UMC, containing links to online surveys. The initial recruitment email was sent out followed by reminder emails, 1 week apart for a total of three to four opportunities to participate.

We were primarily interested in information on work conditions and personal medical health since the start of the pandemic, defined by dates when the first cases of COVID-19 were reported in each state: March 10 for TH and March 5 for UMC. Some questions concerned the most recent week worked since diagnosis of the first case in each state. On June 3, 2020, we distributed 
invitation to TH survey aimed at nurses including advanced nurse practitioners (203) and registered nurses $(4,336)$; at the same time, we distributed invitation to TH survey aimed at physicians to 2,496 active medical staff and 204 physician assistants; all messages were delivered to the recipients. On September 9, 2020, we distributed invitations to the UMC version of the survey to both nurses including nurse practitioners (1518) and physicians (1186). The participation rate was only in the range of $5-10 \%$ (actual counts given in the results).

We used the Hospital Anxiety and Depression Scale (HADS) to measure symptoms of anxiety and depression separately; scores of equal to or above 11 (range 0-21) indicate presence of these conditions but are not equivalent to clinical diagnosis (Zigmond and Snaith, 1983; Bjelland et al., 2002). Higher scores indicated higher chance of having the conditions. The CommunityAcquired Pneumonia Symptom Questionnaire (CAPSym) uses a standard list of symptoms of a wide range of infections to determine the risk of pneumonia, symptomatically close to COVID-19. Lamping et al. (2002) developed and validated the instrument. We used CAP-Sym to determine whether our participants experience symptoms consistent with COVID-19 since the beginning of the pandemic in each state. We evaluated resilience using the two-item Connor Davidson Resilience Scale (CD-RISC2) (Vaishnavi et al., 2007).

In addition to demographic characteristics such as age, marital status, years in profession, gender, educational level, and location of unit and duty assignment, we queried contact with known or suspected COVID19 patients, involvement with aerosol-generating procedures on known and suspected COVID-19 patients such as suspected risk of infection at the time and thus a plausible source of anxiety, belief about having been infected with virus that causes COVID-19, history of anxiety and depression prior to the pandemic (and evidence of exacerbation requiring treatment a year before the pandemic), or history of respiratory and other conditions known at the time, that would place a person at elevated risk due to COVID-19 such as asthma, chronic obstructive pulmonary disease, and emphysema, for example. This was modeled on Canadian Community Health Survey elements (Canada, 2020), and a battery of questions about perceptions (captured on Likert-like scale ranging from 0 to 100 ) of working conditions in the most recent week of work, confidence in work safety (including personal protective equipment (PPE), sources of anticipated support during pandemic, and specific worries. "Worrying" is an established proximal antecedent of generalized anxiety assessed by HADS instrument, as opposed to a more distal "environmental" cause (Newman et al., 2013; Bailey and Wells, 2016). Consequently, we did not adjust for worries in regression models of HADS scores described below, but rather (a) investigated association among worries and HADS for anxiety in principal components analysis and (b) used reported worries descriptively with respect to their correlation with HADS scores. Copies of research instruments are available upon request, but the key questions not present in the cited literature are reported as part of results below.

\section{Statistical analysis}

All calculations were performed in SAS v 9.4 (SAS Institute, Cary, NC). Association of HADS scores for anxiety (HADS A) and depression (HADS D) was examined for each of the covariates of interest in terms of counts of scores $\geq 11$ (referred to as "cases" hereafter) for categorical covariates and Spearman rank correlations for continuous covariates. Bivariate associations of continuous HADS scores with categorical variables were evaluated in Kruskal-Wallis (K-W) tests. We conducted path analyses to determine relationships between HADS scores, belief in having been infected, history of symptoms of pneumonia since start of infection (CAPSym), and belief about contact with COVID-19 patients (PROC CALIS ... method=MLM) (Satorra and Bentler, 1994). All analyses were stratified by discipline (nurse, physician) and study site (TH, UMC); we chose not to pool data to preserve unique features of each site and discipline, as well as knowing that pooling site and discipline data to increase power would deter from the interpretation of the data. Additionally, we elected to conduct stratified analyses instead of pooling data across location and discipline to avoid modeling assumptions that would be involved, such as specific mathematical forms of interaction terms and homogeneity of effect estimates and variances across strata. It must be also considered that the COVID-19 pandemic unfolded on different timescales at the two locations, and we cannot assume that the two healthcare systems are not systematically different, e.g., in safety culture and mitigation measures. Pooling data would exacerbate the issue of multiple comparisons and would allow the larger site and discipline ( $\mathrm{TH}$ and nurses) to dominate pooled data, thereby degrading value of the analysis for the healthcare systems. Multivariable regression models of HADS scores were estimated using binomial regression on TH data only (it proved to be of sufficient size to yield stable regression models that converged; PROC GENMOD). These yielded relative rates (RR) and $95 \%$ confidence intervals (CI) of change in HADS scores in relation to variables that showed evidence of association 
with HADS scores in bivariate analyses, adjusted for each other and plus all demographic variables. Missing values of continuous variables were replaced with means of observed values; there were no missing values for the categorical variables.

\section{Results}

\section{Nurses: demographics, work, and health histories}

Nurses recruited at TH (623) and UMC (61) shared many characteristics in terms of demographics and levels of symptoms of anxiety, depression, and history of episodes of pneumonia since the onset of the pandemic; few of them were tested for COVID-19 (Table 1). Prevalence of anxiety cases ( $>30-39 \%$ ) exceeded that of depression cases by about $12 \%$. Depression and anxiety scores had rank correlation of $0.7(P<0.0001)$ in both groups of nurses.

The enrolled TH nurses were aged 21 to 70 with average of 43 (SD 12) years; they became Registered Nurses between 1975 and 2020, with the mean year of registration being 2003 (SD 12). They had an average HADS anxiety score of 8.7 (SD 4.6)and an average HADS depression score of 5.7 (SD 4.0). Among TH nurses, CAP-Sym scores was on average 12 (SD 19.2) and was weakly correlated with both HADS scores (0.2, $P<0.0001$ ), and CD-RISC2 was on average 6 (SD 1) and inversely related to HADS scores $(-0.4, P<0.0001)$.

The enrolled UMC nurses were aged 25 to 67 with average of 46 (SD 11) years; they became Registered Nurses between 1979 and 2019, with the mean year of registration being 2002 (SD 10). They had an average HADS anxiety score of 9.4 (SD 4.6) and an average HADS depression score of 6.2 (SD 3.9). Among UMC nurses, CAP-Sym scores was on average 22 (SD 21.2) and was positively correlated with HADS scores: 0.3 with anxiety scored $(P=0.04)$ and 0.5 with depression score $(P=0.0002)$. The CD-RISC2 was on average 6 (SD $1)$ and inversely related to HADS scores $(-0.3, P<0.02)$.

Nurses were predominantly female and non-Hispanic white, were married, and had children under 18 years of age living at home; the majority were Registered Nurses (Table 1). We noted evidence of increased cases of anxiety among TH nurses who had recent direct patient contact or were involved in aerosol-generating procedures within a week during pandemic. Nurses we surveyed at UMC exhibited similar patterns.

Among health-related factors, one of the most striking features of the data, consistent across study sites, is the higher rates of anxiety and depression cases among nurses who were unwell for two consecutive days since start of the pandemic and who believed that they were infected (Table 1). History of anxiety and depression, especially among those requiring treatment, was associated with an increased prevalence of both anxiety and depression, but no such pattern was seen for respiratory disease. The patterns of results were similar for both groups of nurses.

\section{Physicians: demographics, work, and health histories}

We recruited 135 physicians at TH and 50 at UMC (50). Physicians in TH and UMC samples were mostly nonHispanic white and married, with about half reporting that they had children under 18 living at home (Table 2); sample of TH physicians was gender-balanced, but there were more men in the UMC sample. Just as with nurses, the majority were not tested for COVID-19. As with nurses, among physicians the prevalence of anxiety cases exceeded that of depression cases. Depression and anxiety scores had rank correlation of $0.7-0.8(P<0.0001)$ among physicians.

Physicians were somewhat older than nurses: TH physicians were on average 49 (SD 12) years of age, UMC physicians-52 (SD 11). The physicians were licensed to practice medicine between 1970 and 2019, medians in the late 1990s. Physicians had lower HADS and CAP-Sym scores than nurses in their respective healthcare systems, as detailed below.

TH physicians had an average HADS anxiety score of 7.1 (SD 4.0) and an average HADS depression score of 4.2 (SD 3.4). Among TH physicians, CAP-Sym scores was on average 5.6 (SD 12.8) and correlated with both HADS scores $(r=0.2, P=0.01)$. The CD-RISC2 was on average 7 (SD 1) and inversely related to HADS scores $(r=-0.3, P<0.001)$.

UMC physicians had lower HADS scores than their TH colleagues. Specifically, their average HADS anxiety score was 5.4 (SD 3.9) and their average HADS depression score was 3.9 (SD 4.0). Among UMC physicians, CAP-Sym scores was on average 12.4 (SD 17.4), higher than at TH; it was not correlated with HADS scores $(r=0.1, P>0.4)$. The CD-RISC2 was on average 7 (SD $1)$ and inversely related to HADS scores for anxiety $(r=-0.6)$ and depression $(r=-0.5)(P<0.0001)$.

The belief that physicians were infected with COVID19 was associated with elevated rates of anxiety and depression cases among all physicians. Having been unwell for two consecutive days since the start of the pandemic likewise was associated with higher HADS scores among TH but not UMC physicians. Unlike with nurses, there appears to be no evidence of an increase in cases of anxiety among $\mathrm{TH}$ physicians who had recent direct patient 


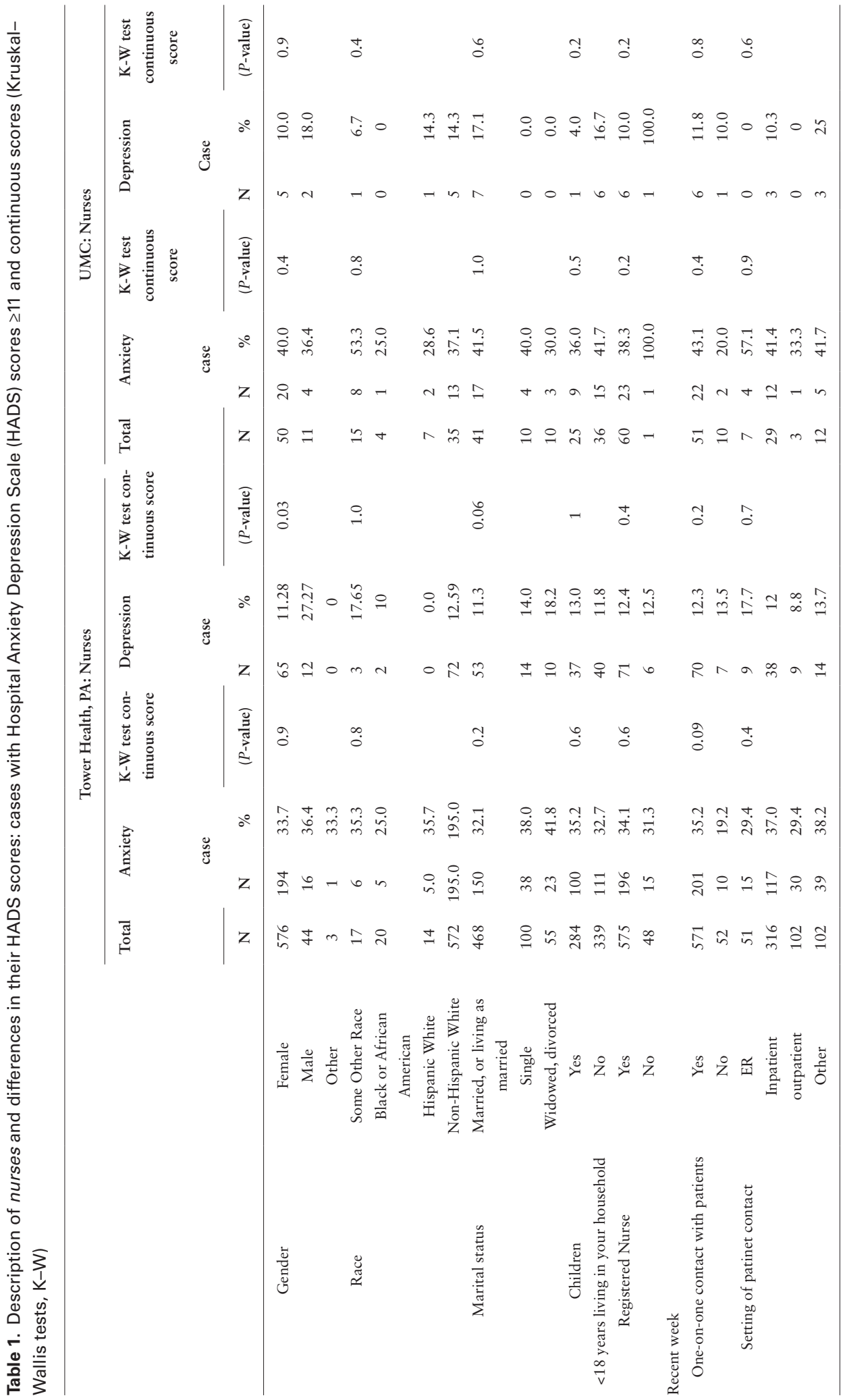




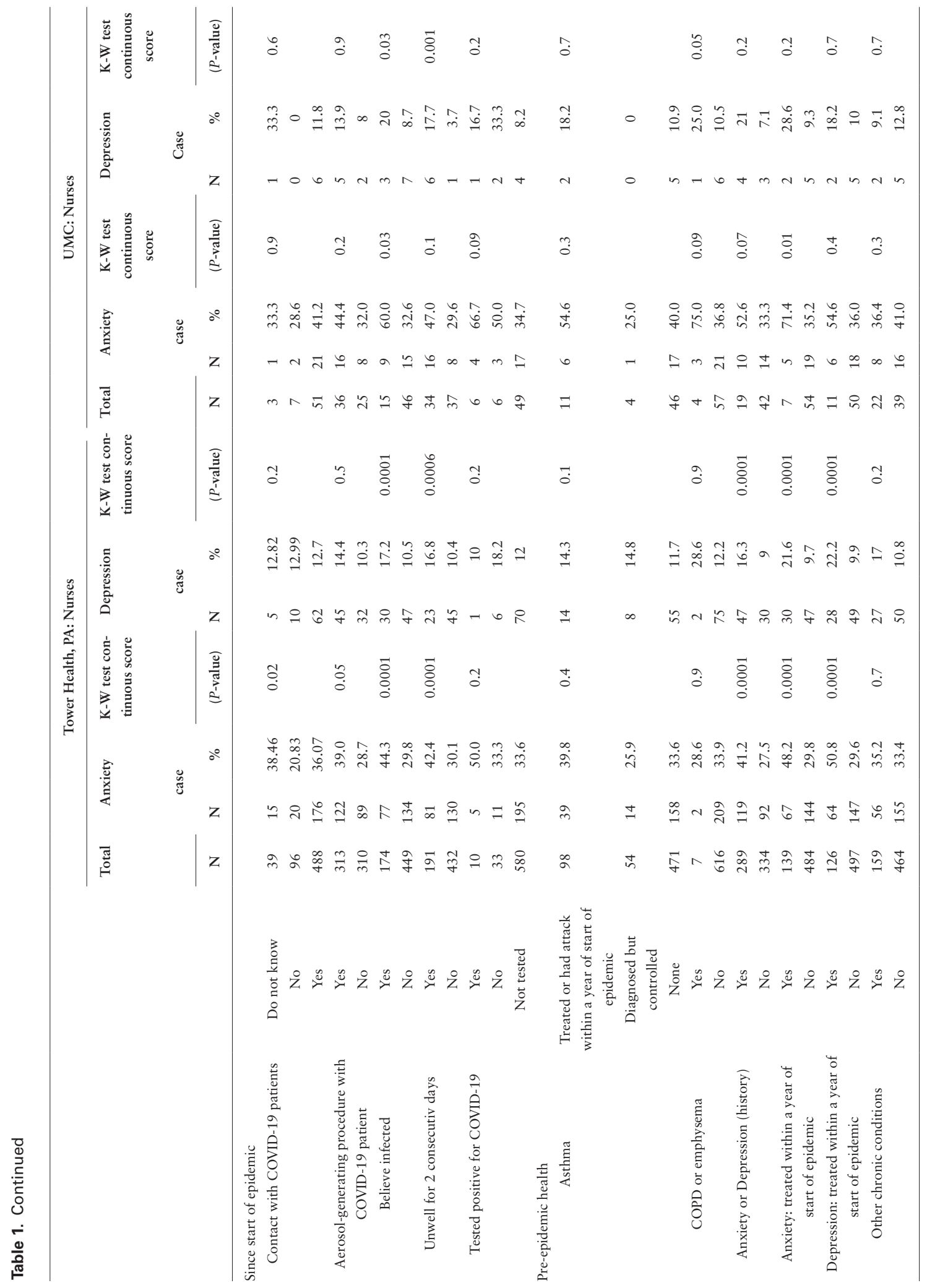




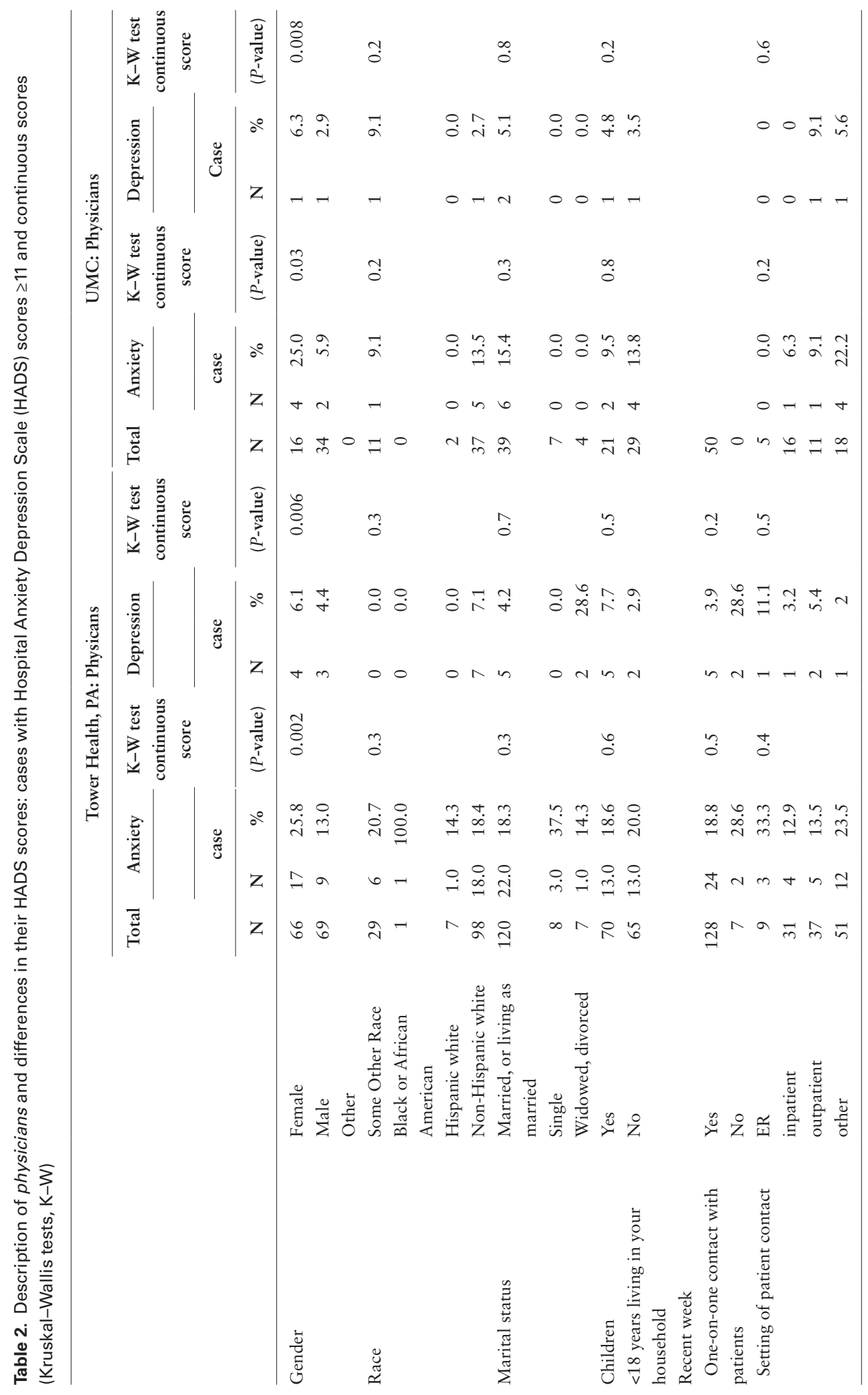




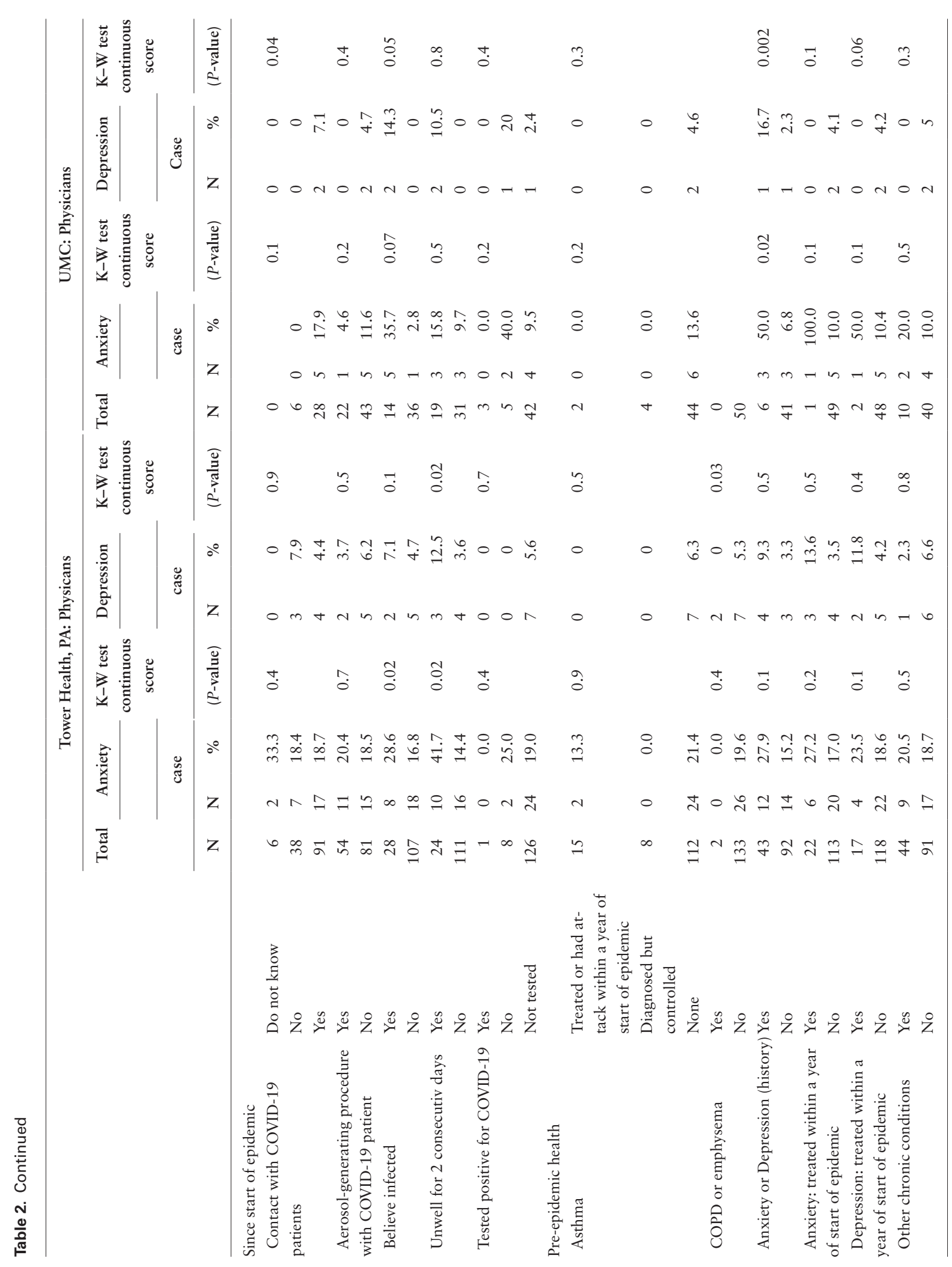


contact during the most recent week of work; all UMC physicians had such patient contact. There was no evidence of association of HADS scores with having been involved in aerosol-generating procedures during pandemic. History of anxiety and depression, but not other conditions, as with nurses, were related to higher numbers of cases of anxiety and depression.

\section{Concerns and perceptions}

Bivariate analysis of concerns and perception in relation to HADS scores is presented in Table 3 for nurses and in Table 4 for physicians.

When asked to record "perception of work during recent week of epidemic" on a Likert-like scale, nurses tended to agree that "hours of work," "tasks," and "patient make-up" did not change, with median scores of at or above 50 (Table 3). On the other hand, both patients and co-workers were perceived as more stressed, with most tending to disagree that this was so, with median scores below midpoint of the scale. All these perceptions were negatively correlated with HADS scores among TH nurses, with the lower levels of anxiety and depression associated with lower perceived stress among co-workers during recent week of work. Among UMC nurses, only the perception that working hours were about the same during recent week of work was associated with reduced HADS scores. Among physicians, we observed similar patterns to those among nurses, except in the sample of UMC physicians (Table 4).

Confidence in working with COVID-19 patients with respect to PPE use and sufficient staffing was high among nurses, with median scores above middle of the scale and confidence in knowledge of how to use of PPE near the top of the scale (Table 3). Confidence in "sufficient staff to do the job safely" was most strongly inversely related to anxiety and depression. Among physicians, confidence in PPE and staffing was likewise high, but its inverse association appeared to be limited to UMC physicians (Table 4).

Nurses at TH tended to report finding strong support only among immediate family, colleagues or co-workers, or a senior colleague or mentor, with median scores at or above the middle of the scale (Table 3). Among TH nurses, greater confidence in any source of support was associated with reduction of HADS scores, except for the reverse trend with reports of finding support from the trade union at TH. Nurses at UMC tended to believe that they would find stronger support among immediate family, colleagues or co-workers, a senior colleague or mentor, their immediate organization, and employer, with median scores at or above the middle of the scale. Only support from these sources was associated with lower HADS scores among UMC nurses. On average, nurses did not expect to find support from municipal department of public health, State Boards of Nursing, and trade unions.

Among physicians, the dominant reported sources of support were the same as among nurses, with reports of the perceived strongest support from immediate family (average scores $>80 / 100$ ) and perception of American Medical Association being the least likely source of support (average scores around 20/100) (Table 4). Perception of stronger support from colleagues and co-workers was associated with lower HADS scores in both groups of physicians. Lower depression scores were related to perception of stronger support from religious communities among physicians. There was a tendency for perception of stronger support from any source other than immediate family, to be linked to lower HADS scores among UMC physicians. The pattern was different for TH physicians for whom only perception of stronger support from family appeared to be protective.

We inquired about "worries about the COVID-19 epidemic" and captured it on a Likert-like scale. As illustrated in Table 5, by far the greatest worry was of infecting one's family, followed by worry about being infected oneself. Worries related to performance of professional duties were relatively less prominent. These patterns were consistent across sites and professional groups. The strongest of the associations with HADS scores, for each site and profession, was a worry that the person will fail themselves and their family. HADS scores for anxiety and all responses about worries were associated with one latent component in principal components analysis accounting for the majority of common variance (e.g., 50\% among TH nurses, the most informative of our samples, and $43 \%$ for $\mathrm{TH}$ physicians); only one latent component was suggested by the scree plots (details not shown).

\section{Multivariable models of HCWs at Tower Health}

Adjusted effect estimates of covariates examined above for TH cohort are summarized in Table 6; effect estimates for perceptions is shown per 25 units (about one SD). Similar analyses for UMC did not produce stable models and therefore are not reported, although their findings largely agree with patterns seen at TH. After controlling for all other evaluated circumstances, the higher pneumonia symptom score (CAP-Sym) over a two-day period since diagnosis of the first COVID-19 case in the state was the most consistent predictor of higher risk of anxiety and depression across the two professions. Plots of observed and predicted HADS scores in relation to CAPSym suggest good model fit (Supplementary Appendix 







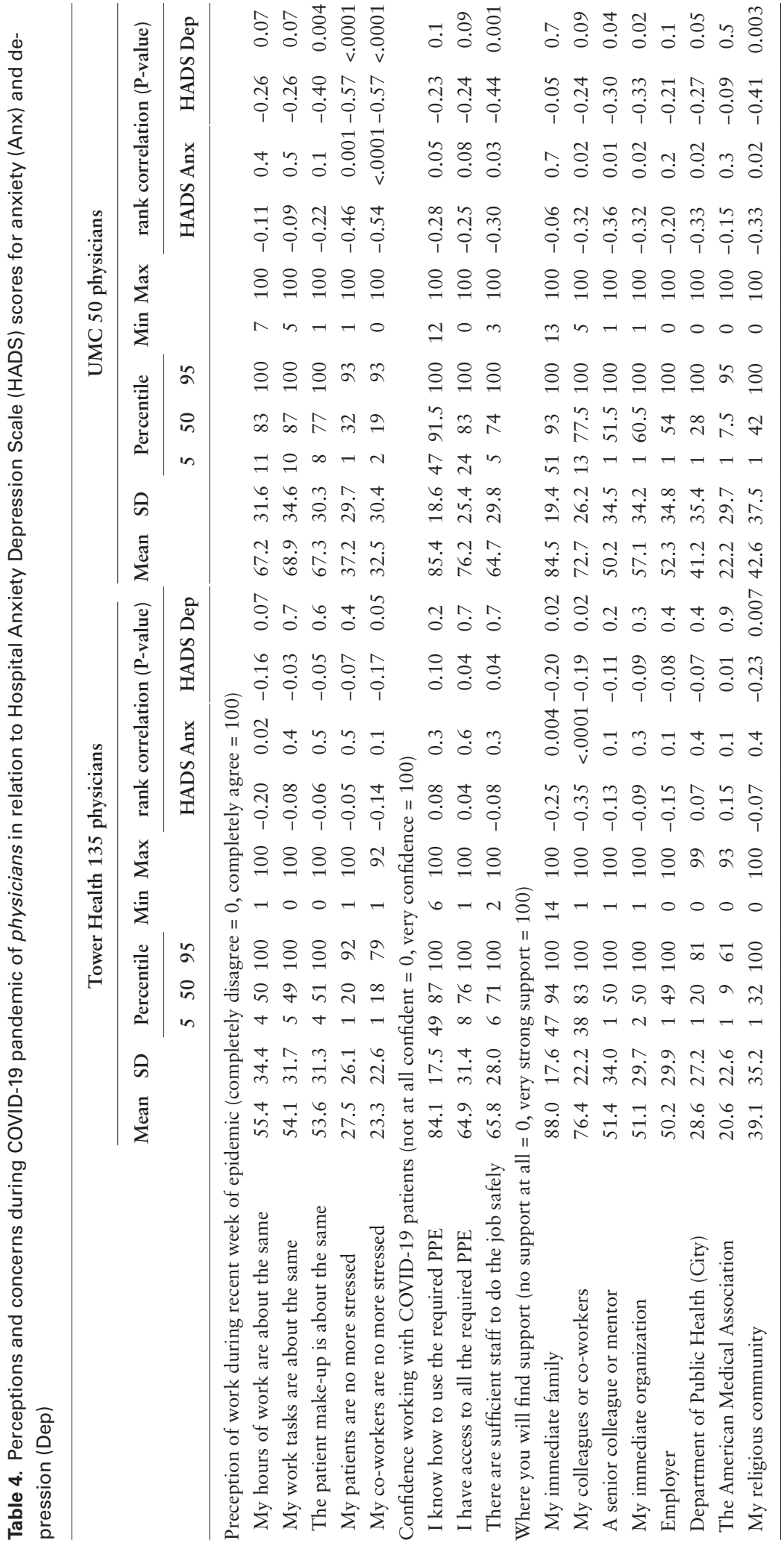




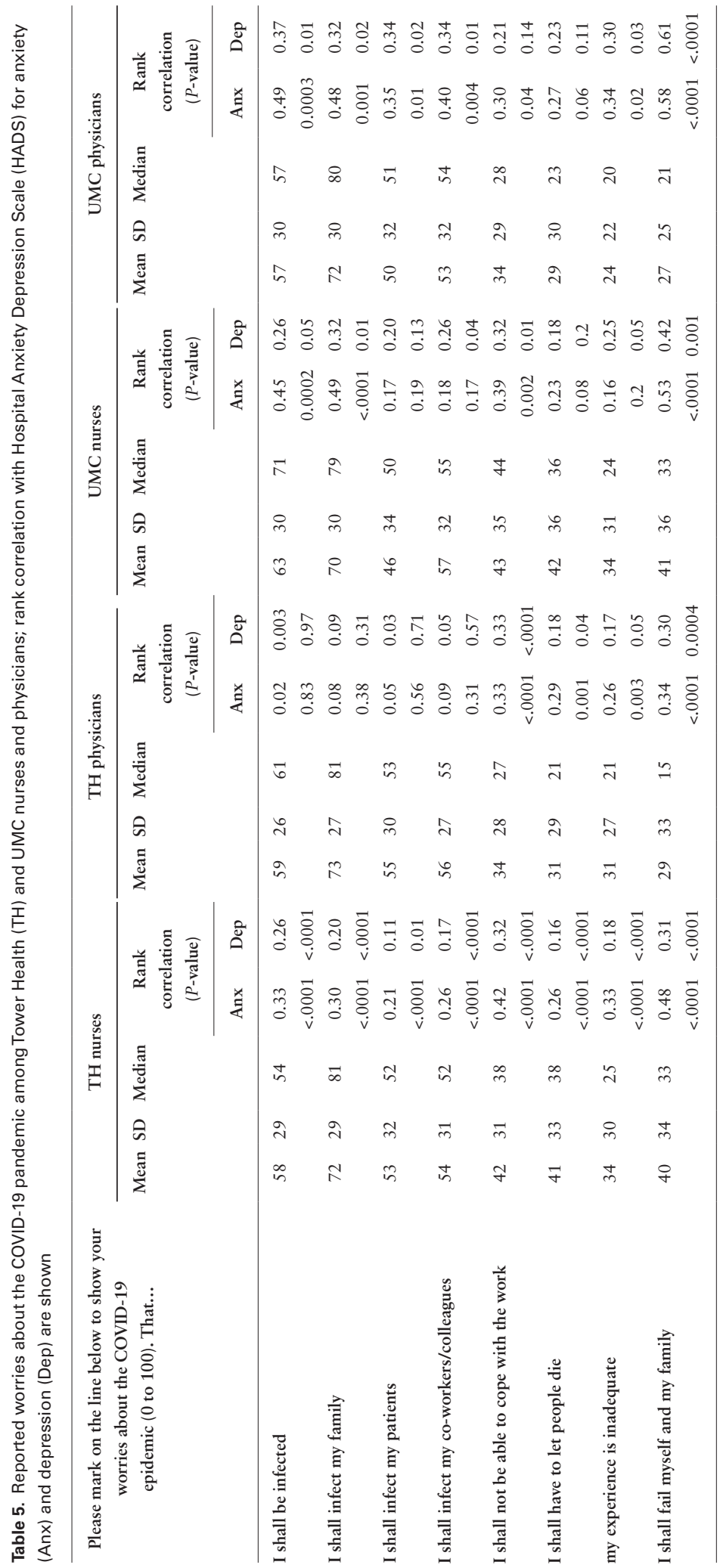




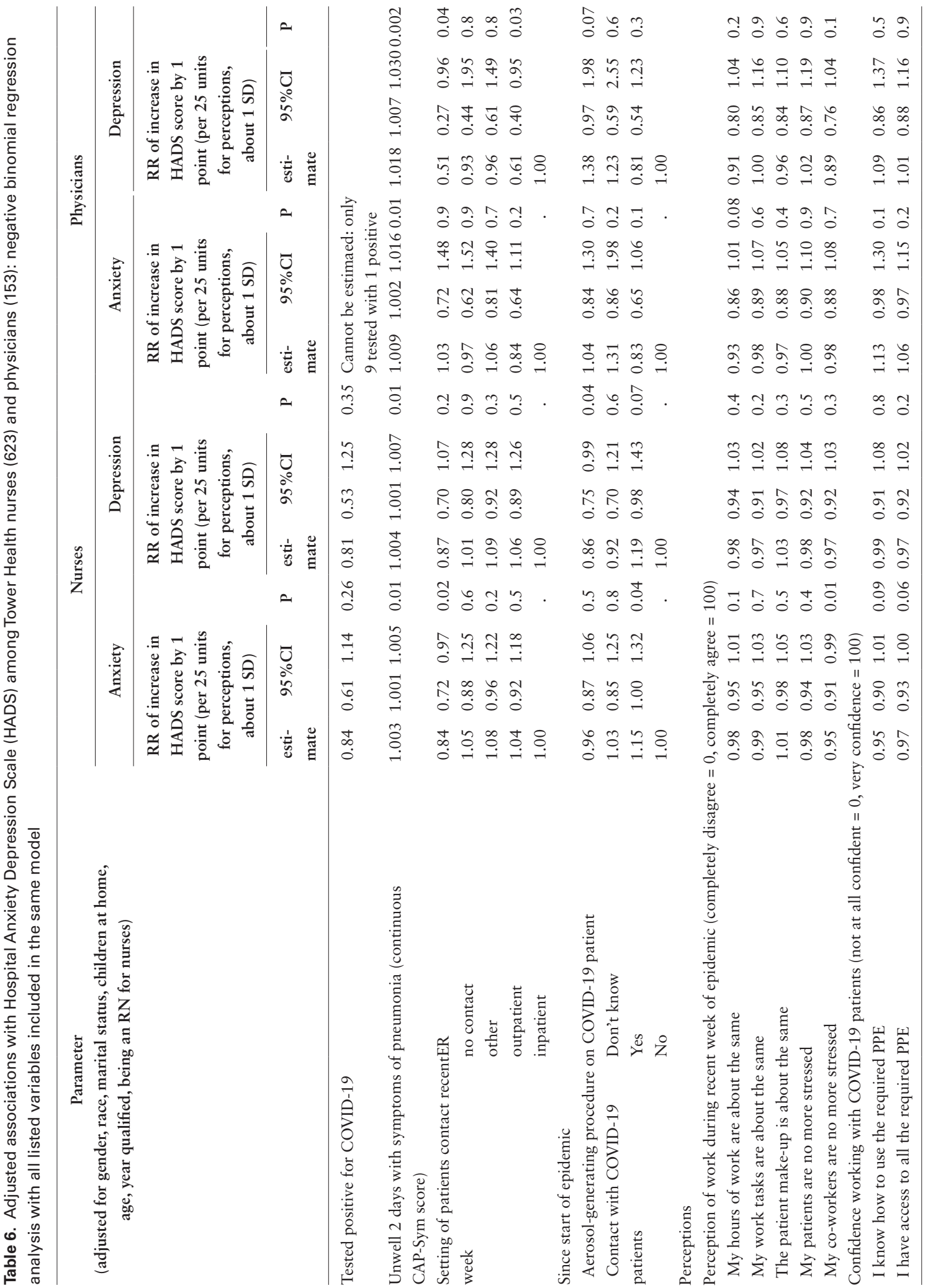




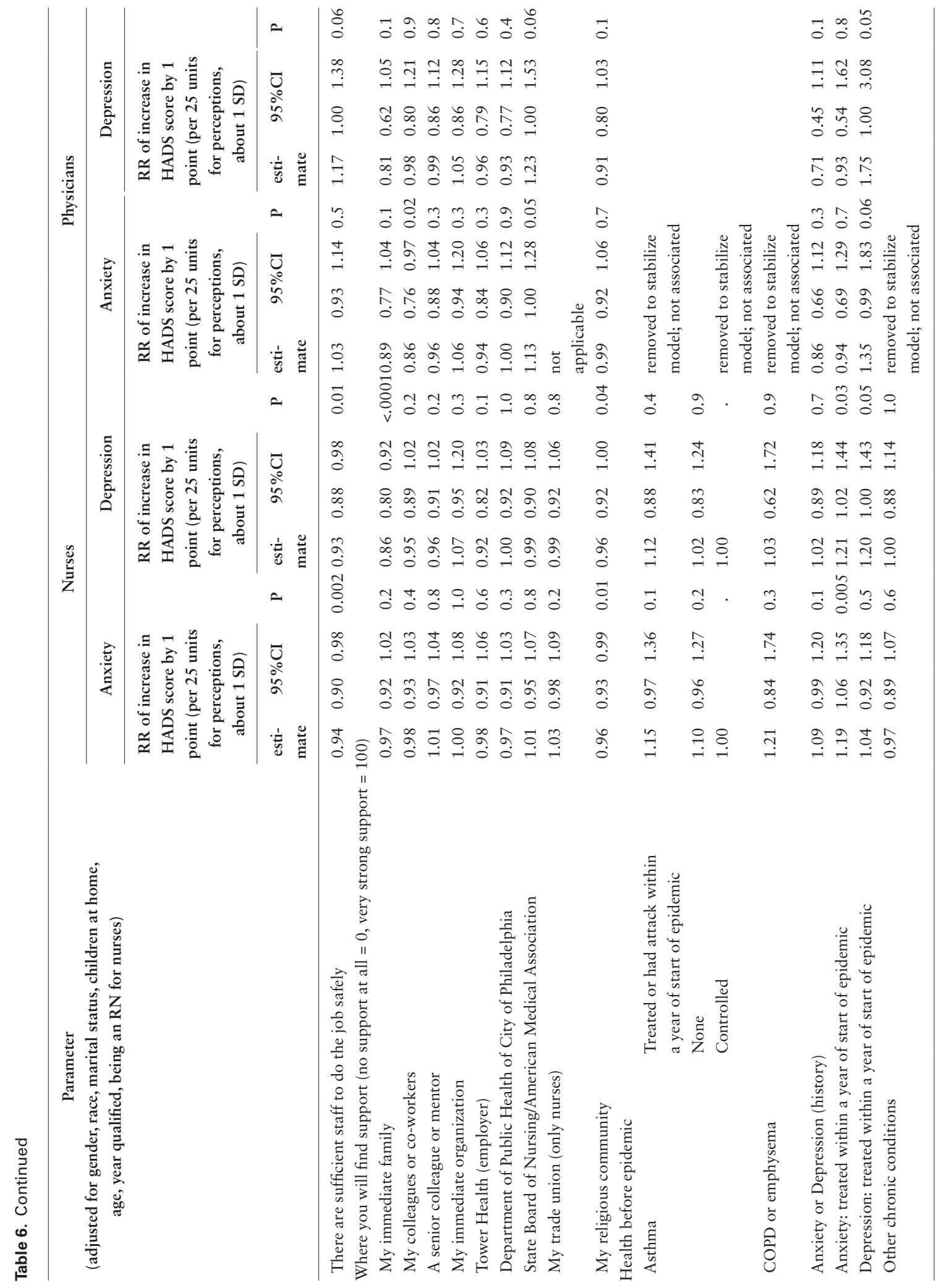


A, available at Annals of Work Exposures and Health online). This factor was correlated with belief in having been infected, which, being seen by us as an intermediate on the pathway towards anxiety and depression, was not forced into regression models; belief in having been infected is instead considered as pathway analysis below.

In adjusted analyses, nurses and physicians who recently encountered patients in emergency departments (ER) showed evidence of reduced risk of symptoms of anxiety and depression, respectively, relative to those who treated patients in the inpatient setting. Physicians who encountered patients in the outpatient settings were likewise less likely to show symptoms of depression relative to those who worked in inpatient settings. There was no evidence of other associations with setting of recent patient contact.

Having knowledge of any contact with COVID-19 patients was associated with, on average, $20 \%$ higher anxiety and depression scores in nurses relative to those who reported no such contact; no such associations were evident among physicians, except for a suggestion of reduced anxiety among physicians who thought that they had had contact with COVID-19 patients relative to those who did not (RR $0.83,95 \%$ CI: $0.65,1.06$ ). There was also some evidence that not knowing whether physicians encountered COVID-19 patients was a cause for anxiety (RR 1.31, 95\% CI: 0.86, 1.98). After allowing for knowledge of contact with COVID-19 patients, the reports of having performed aerosol-generating procedure on COVID-19 patients was not associated with anxiety but appeared to be related to reduced HADS scores for depression among nurses (RR 0.86, 95\% CI: $0.75,0.99)$, with the opposite effect among physicians (RR $1.38,95 \%$ CI: 0.97, 1.98).

Among perceptions of work during most recent week of the pandemic, reports of working hours remain the same; co-workers perceived no additional stress which was associated with lower HADS scores, the strongest effect estimate was with lower anxiety scores among nurses who reported that their co-workers were "no more stressed" (RR 0.95, 95\% CI: 0.91, 0.99). Believing that there was enough staff to do the job safely was associated with both reduced anxiety and depression scores among nurses; anxiety was also lower among nurses who reported that they know how to use PPE and have access to it (with no such effect on the depression score). There was a suggestion that physicians who were confident in how to use PPE were also more anxious (RR $1.13,95 \%$ CI: $0.98,1.30$ ) and that those who were confident in having sufficient staff to do the job safely tended to be more depressed (RR 1.17, 95\% CI: 1.00, 1.38). Nurses and physicians who reported that they will have strong support from their families showed lower scores of symptoms of anxiety and depression, having allowed for all other factors in the analysis. Physicians who reported that they will find strong support from the American Medical Association were more likely to show symptoms of anxiety and depression; there was no analogous effect among nurses with respect to the State Board. Nurses who reported that they will find support in their religious community were less anxious and depressed; there was a suggestion of similar effect among physicians, especially for depression.

Among nurses, being treated for anxiety up to a year before the pandemic was independently associated with HADS scores, with additional positive association between history of treatment for depression within a year of start of the pandemic and depression score. Among physicians, being treated for depression up to a year before the pandemic was associated with both higher anxiety and depression scores. No other elements of recorded medical histories appeared to independently relate to HADS scores.

Accounting for measure of resilience did not materially alter the results despite its independent inverse association with anxiety and depression (details not shown).

\section{Path analyses}

Results of path analyses for anxiety are summarized in Figs. 1 and 2, excluding persons who tested positive for COVID-19 did not affect estimated associations. We did not examine all possible causal pathways, but merely estimated associations posited a priori. We estimated that a belief that a person was infected with COVID19 ("Do you have reason to believe that you may have been infected with the COVID-19 virus?": Yes/No) is directly related to higher HADS anxiety scores at both sites and professional groups. Likewise, the higher pneumonia (CAP-Sym) score was positively related to belief in having been infected. Data from TH revealed evidence of both the direct effect of CAP-Sym on anxiety and that mediated by belief in having been infected (Fig. 1). There was a positive association between CAP-Sym and contact with COVID-19 patients. Path analyses with depression score as outcome were different from those for anxiety in only two respects: there was no evidence that belief in having been infected was associated with HADS depression scores among UMC nurses and TH physicians, and there was evidence of direct effect of CAP-Sym on depression scores among UMC nurses. The results of these analyses are given in Supplementary Figs. S1-S4 (Supplementary Appendix B, available at Annals of Work Exposures and Health online). Path analysis on data pooled across sites and disciplines yielded evidence of all hypothesized pathways (not shown). 
Tower Health Nurses: paths with $p \leq 0.05$ marked by *

Standardized Parameter Estimates

All/Not tested positive for COVID-19

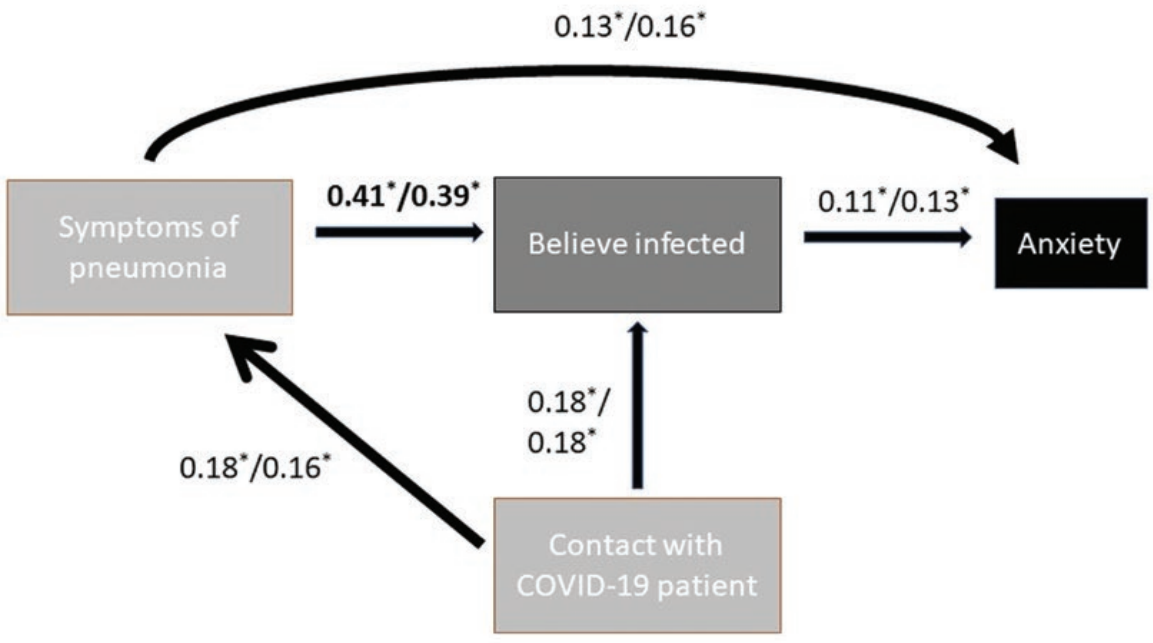

Tower Health Physicians: paths with $p \leq 0.2$ marked by \# paths with $p \leq 0.05$ marked by *

Standardized Parameter Estimates

All/Not tested positive for COVID-19

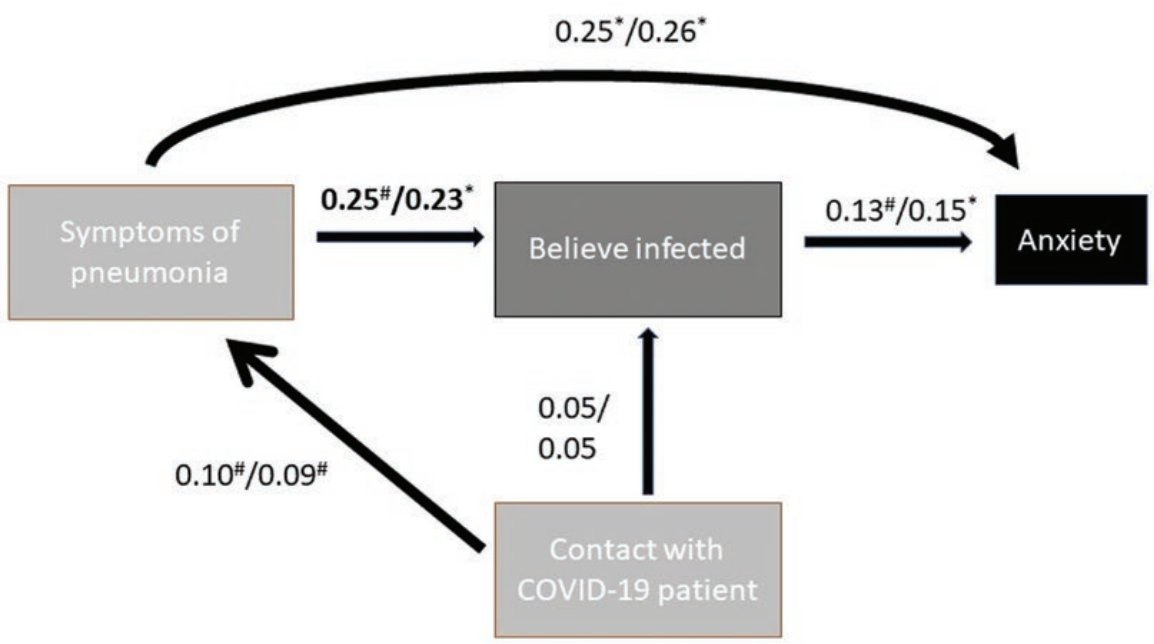

Figure 1. Pathways connecting Hospital Anxiety and Depression Scale anxiety score to symptoms of pneumonia (CAP-Sym) through belief of having been infected with virus that causes COVID-19, with consideration of contact with COVID-19 patients, among healthcare workers from Tower Health, PA (623 nurses [top] and 135 physicians [bottom]).

\section{Discussion}

We observed that about a third of nurses and physicians showed symptoms of anxiety or depression, which is similar, for the comparable time period, to findings by Czeisler et al. (2020) for the USA as the whole, but lower than for the self-identified "essential workers" $(42 \%)$.
Differences in outcome assessment instruments make exact comparison problematic but HADS scores that we observed among HCWs are clearly above normative values established in the UK (Breeman et al., 2015), with median normative scores for anxiety in 5-6 range and for depression about 3 . We observed average scores 
UMC Nurses: paths with $p \leq 0.2$ marked by\#

p $\leq 0.05$ marked by*

Standardized Parameter Estimates

All/Not tested positive for COVID-19

$0.16 / 0.11$

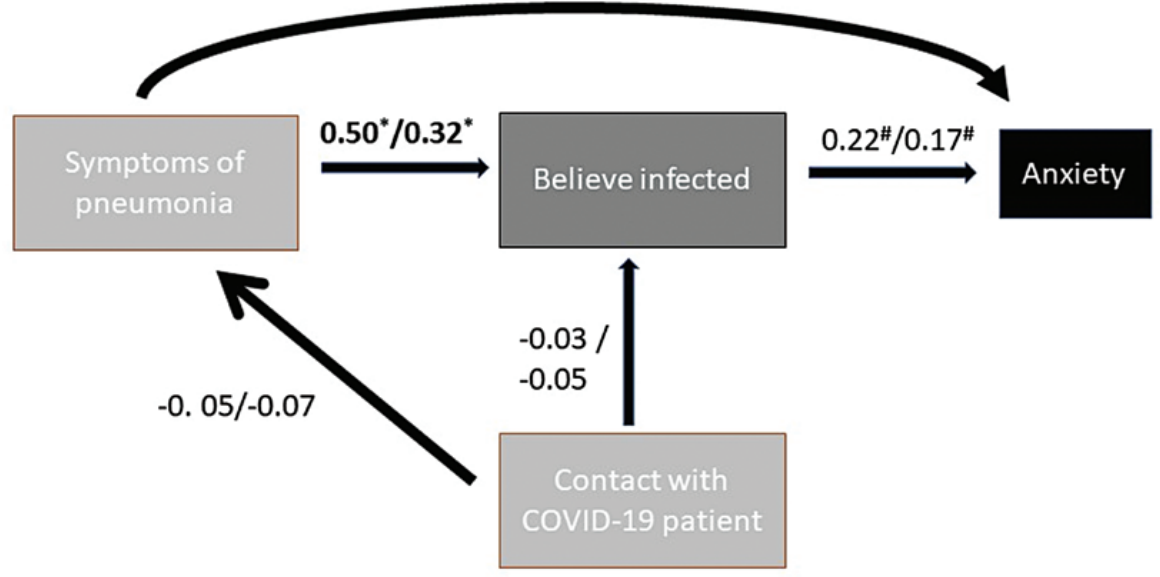

UMC Physicians: paths with $\mathrm{p} \leq 0.05$ marked by *

Standardized Parameter Estimates

All/Not tested positive for COVID-19



Figure 2. Pathways connecting Hospital Anxiety and Depression Scale anxiety score to symptoms of pneumonia (CAP-Sym) through belief of having been infected with virus that causes COVID-19, with consideration of contact with COVID-19 patients, among healthcare workers from the University Medical Center (UMC), Nevada, LV (61 nurses [top] and 50 physicians [bottom]).

greater than those reported for Canadian firefighters at the time they faced devastating Fort McMurray fires and were seen to develop elevated rates of post-traumatic stress disorder 3 years later (Cherry et al., 2020). Specifically, we see evidence of greater than expected levels of anxiety and depression in nurses from both healthcare systems and physicians from TH but not from UMC. This is likely an under-estimate of a higher prevalence of mood disorders among HCWs in the two healthcare systems. A cross-sectional study design limited recruitment to active employees, excluding those who are too ill to work. However, we cannot exclude the 
possibility of our estimates being upwardly biased by the survey adding suggestion to participants who experience mental health difficulties compared to those who do not have that medical history.

We evaluated resilience via the two-item Connor Davidson Resilience Scale but adjustment for it did not alter the results, suggesting that confounding by variation in "'bounce-back' and adaptability" in our sample is unlikely. The mean resilience scores were typical of the US general population among physicians (7 out of maximum of 8 ), but disturbingly, these scores were in the range of family medicine and psychiatric outpatients among nurses (around 6) (Vaishnavi et al., 2007). This is concordant with higher levels of symptoms of anxiety and depression among nurses, reinforcing the suggestion that the mental health of nurses is more severely affected by the COVID-19 pandemic than that of physicians in the studied settings. It is debatable whether adjustments for resilience, which is a personality trait, should be made, as personality can be affected over time by working conditions; however, the adjustment had no impact on the results in these populations so is not reported.

The most often identified worries among nurses and physicians, were that of COVID-19 infection transmitted to the HCW and their family, with far fewer worries about performance of professional duties. Apprehension of failure of one's own expectations of oneself and that of their family was the strongest correlate of anxiety and depression. Although it is tempting to speculate that addressing these specific worries through mental health support services may have alleviated the burden of symptoms of anxiety and depression overall, it is not clear this would have alleviated either anxiety or depression.

Belief in having been infected with COVID-19 (whether one tested positive for the virus or not) emerged as a prominent cause of anxiety and depression, related more to history of symptoms known to HCWs to be consistent with COVID-19 at the time when testing may have been both limited and unreliable (not trusted), rather than actual exposure to infected patients. Among work-related factors that we identified as protective against anxiety and depression were as follows: (i) having confidence in competent use and access to PPE, (ii) maintaining usual working hours, and (iii) being surrounded by colleagues who were both sufficient in numbers and not stressed. Having support of immediate family and religious communities lessened anxiety and depression after accounting for other factors but any support was beneficial, although it was mostly believed that it will come from personal connections rather than professional bodies. There was some evidence that HCWs in emergency departments were less anxious and depressed and no clear evidence that involvement in aerosol-generating procedures on the infected patients was important per se.

Strengths of our work include the use of HADS scores, which are more precise than commonly employed alternatives in large-scale epidemiologic studies (Rose and Devine, 2014) such as Patient Health Questionnaire (PHQ-4) (Czeisler et al., 2020). However, it would have been desirable to employ measure of mood disorders that is directly comparable to the literature emerging from China and Canada. All previous work employed ad hoc questions of unknown psychometric properties to assess symptoms of COVID-19, while we used a validated questionnaire that captured symptoms by noting that they are consistent with community-acquired pneumonia. Thus, our analysis is less prone from bias due to errors in key outcomes and exposures. Our work involved use of a well-defined sampling frame, not a convenience sample, making it less prone to bias from unmeasured confounding factors related to participation and outcomes.

Perception and concern questions were developed specifically for our study, and we did not have the opportunity, due to the punishing timetable imposed by the pandemic, to assess their reliability and validity. However, we are reassured by the fact that they yielded expected associations but acknowledge that bias from residual differential measurement error is possible. Differential measurement error may have arisen if, plausibly, persons more distressed by experience during pandemic were more likely to participate and made a greater effort in accurately responding to perceptions and concerns questions. Such selection mechanism may bias both internal and external validity of our findings and we are not able to address them quantitatively due to lack of information on even the demographics of non-participants. These concerns are aggravated by participation rate of $5-10 \%$. This places generalizability of our findings in question, a matter aggravated by the fact that we do not have access to information on differential recruitment into our survey. However, our sample size is sufficient to yield robust inference (with adjustment for multiple factors via regression modeling) for the larger of the samples at TH and is informative of the situation experienced by selected participants at UMC. External validity of our findings is undermined by not including representative range of HCWs, such as licensed practical nurses, physician assistants, physical therapists, occupational therapists, respiratory therapists, Certified Nurses' Aides, to name a few. However, existence of some concordance among 
studied professions among HCWs, mostly registered nurses and medical doctors, leaves us optimistic that some of the patterns we observe may be informative of the experience of all healthcare workers, the notion that is supported by our findings being largely in agreement with those from other jurisdictions.

There are likely factors related to working conditions (and their perceptions) and mood disorders that were not captured in our data, like insomnia and substance use, that could have confounded observed associations, but they may also be mediators of the effects of psychological and other working conditions on mental health, i.e., not sources of bias in our analysis. However, we believe that we captured major confounders among our demographic and health-related variables, such that the risk of latent cofounding is reduced though regression adjustment for TH nurses and physicians. Measured confounders had little impact on direction and magnitude of the associations with pneumonia symptoms and associations with perceptions of PPE and working conditions, reassuring us in the robustness of these observations. We controlled for pre-existing mental health issues in isolating pandemic-related causes of anxiety and depression, further reducing the chance of bias in the results.

There was some heterogeneity in findings among two study sites, but they may be either due to chance or local peculiarities of healthcare systems' and States response to the pandemic. Our findings of impact of work organization on anxiety and depression (staffing issues, hours of work, and perceived co-worker stress) may be related to the role of safety climate or culture in moderating impact of the pandemic on work-induced mental health issues(Dollard and Bakker, 2010; Rickard et al., 2012). It would be helpful to formally evaluate this notion within appropriate theoretical frameworks that can inform workplace interventions (Dollard et al., 2019). It is likely that application of the self-determination theory can be helpful in accelerating and sustaining compliance with any workplace changes that would need to be made (Burstyn et al., 2010), especially under the extreme pressures of a pandemic. One is tempted to speculate that development of stronger safety culture and autonomy-supportive management is an excellent step in preparedness of emergencies in any setting, including healthcare.

There were some differences in level of stress and anxiety and their correlates between nurses and physicians. This may be in part attributed to patient contact being typically longer and more intimate for nurses. However, common themes also emerged, specifically related to pathway by which experience of pneumonia symptoms, known contact with COVID-19 patients, and belief in having been infected related to symptoms of anxiety and depression. There was no evidence that differences in anxiety and depression seen between nurses and physicians are explained by gender alone: results of regression analysis of pooled data adjusting for gender, discipline and site are not shown but revealed increased risk among nurses relative to physicians after accounting for gender.

\section{Conclusion}

We conclude that the levels and correlates of anxiety and depression among physicians and nurses in two US healthcare systems reveal that their experiences are like those of their colleagues around the world. It is not our place to speculate about specific mitigation measures that healthcare systems may wish to pursue to alleviate the burden of anxiety and depression among healthcare workers. Instead, we trust that our findings will help develop such measures and underscore the need to help nurses and physicians bear the psychological burden of combating COVID-19 pandemic and similar events in the future.

\section{Supplementary data}

Supplementary data are available at Annals of Work Exposures and Health online.

\section{Conflict of interest}

We declare no conflicts of interest.

\section{Acknowledgments}

The authors are deeply indebted to all the participants who responded to survey while learning to live and work under disruptions precipitated by the pandemic. Staff of the two healthcare systems provided invaluable support in deploying the surveys. The questionnaire was developed for the Canadian inter-provincial study of COVID-19 in health care workers and was made available for the present study by the Principal Investigator, Dr. Nicola Cherry at the University of Alberta.

\section{Data availability statements}

The data underlying this article cannot be shared publicly to protect the privacy of individuals that participated in the study. De-identified summaries of the data necessary to either reproduce specific results, or illuminate questions not addressed in the paper, will be shared on reasonable request to the corresponding author. 


\section{References}

Bailey R, Wells A. (2016) Is metacognition a causal moderator of the relationship between catastrophic misinterpretation and health anxiety? A prospective study. Behav Res Ther; 78: 43-50.

Barrett ES, Horton DB, Roy J et al. (2020) Prevalence of SARSCoV-2 infection in previously undiagnosed health care workers in New Jersey, at the onset of the U.S. COVID-19 pandemic. BMC Infect Dis; 20: 853.

Bjelland I, Dahl AA, Haug TT et al. (2002) The validity of the Hospital Anxiety and Depression Scale. An updated literature review. J Psychosom Res; 52: 69-77.

Breeman S, Cotton S, Fielding S et al. (2015) Normative data for the hospital anxiety and depression scale. Qual Life Res; 24: 391-8.

Burstyn I, Jonasi L, Wild TC. (2010) Obtaining compliance with occupational health and safety regulations: a multilevel study using self-determination theory. Int J Environ Health Res; 20: 271-87.

Canada S. (2020) Canadian Community Health SurveyAnnual component (CCHS). Book Canadian Community Health Survey-Annual component (CCHS), City.

CDC. (2020) Characteristics of health care personnel with COVID-19 - United States, February 12-April 9, 2020. MMWR Morb Mortal Wkly Rep; 69: 477-81.

Cherry N, Galarneau JM, Melnyk A, Patten S. (2020) Prevalence of mental ill-health in a cohort of first responders attending the fort mcmurray fire: prevalence de mauvaise sante mentale dans une cohorte de premiers intervenants dans l'incendie de fort mcmurray. Can J Psychiatry; 706743720974824.

Chong MY, Wang WC, Hsieh WC et al. (2004) Psychological impact of severe acute respiratory syndrome on health workers in a tertiary hospital. Br J Psychiatry; 185: 127-33.

Czeisler MÉ, Howard ME, Robbins R, Barger LK, FacerChilds ER, Rajaratnam SMW, Czeisler CA. (2020) COVID-19: public compliance with and public support for stay-at-home mitigation strategies. medRxiv; 2020.04.22.20076141.

Dollard MF, Bakker AB. (2010) Psychosocial safety climate as a precursor to conducive work environments, psychological health problems, and employee engagement. J Occup Organ Psychol; 83: 579-99.

Dollard M, Dormann C, Idris MA. (2019) Psychosocial safety climate: a new work stress theory and implications for method. In Dollard M, Dormann C, Idriss A, editors. Psychosocial safety climate: a new work stress theory. Cham, Switzerland: Springer International Publishing.

Firew T, Sano ED, Lee JW et al. (2020) Protecting the front line: a cross-sectional survey analysis of the occupational factors contributing to healthcare workers' infection and psychological distress during the COVID-19 pandemic in the USA. BMJ Open; 10: e042752.

Guo J, Liao L, Wang B et al. (2020) Psychological Effects of COVID-19 on Hospital Staff: A National Cross-Sectional
Survey of China Mainland. Preprints with The Lancet (SSRN) [serial online] 2020.

Lai J, Ma S, Wang Y et al. (2020) Factors associated with mental health outcomes among health care workers exposed to coronavirus disease 2019. JAMA Netw Open; 3: e203976.

Lamping DL, Schroter S, Marquis P et al. (2002) The community-acquired pneumonia symptom questionnaire: a new, patient-based outcome measure to evaluate symptoms in patients with community-acquired pneumonia. Chest; 122: 920-9.

Lancee WJ, Maunder RG, Goldbloom DS; Coauthors for the Impact of SARS Study. (2008) Prevalence of psychiatric disorders among Toronto hospital workers one to two years after the SARS outbreak. Psychiatr Serv; 59: 91-5.

Lee AM, Wong JG, McAlonan GM et al. (2007) Stress and psychological distress among SARS survivors 1 year after the outbreak. Can J Psychiatry; 52: 233-40.

Maunder RG, Lancee WJ, Rourke S et al. (2004) Factors associated with the psychological impact of severe acute respiratory syndrome on nurses and other hospital workers in Toronto. Psychosom Med; 66: 938-42.

Newman MG, Llera SJ, Erickson TM et al. (2013) Worry and generalized anxiety disorder: a review and theoretical synthesis of evidence on nature, etiology, mechanisms, and treatment. Annu Rev Clin Psychol; 9: 275-97.

Pappa S, Ntella V, Giannakas T et al. (2020) Prevalence of depression, anxiety, and insomnia among healthcare workers during the COVID-19 pandemic: a systematic review and meta-analysis. Brain Behav Immun; 88: 901-7.

Preti E, Di Mattei V, Perego G et al. (2020) The psychological impact of epidemic and pandemic outbreaks on healthcare workers: rapid review of the evidence. Curr Psychiatry Rep; 22: 43.

Rebmann T. (2020) COVID-19 in Non-Acute Care Settings: HardEarned Lessons from Two APRNs on the Frontlines [Webinar]. Book COVID-19 in Non-Acute Care Settings: Hard-Earned Lessons from Two APRNs on the Frontlines [Webinar], City.

Rickard G, Lenthall S, Dollard M et al. (2012) Organisational intervention to reduce occupational stress and turnover in hospital nurses in the Northern Territory, Australia. Collegian; 19: 211-21.

Rose M, Devine J. (2014) Assessment of patient-reported symptoms of anxiety. Dialogues Clin Neurosci; 16: 197-211.

Satorra A, Bentler PM. (1994) Corrections to test statistics and standard errors in covariance structure analysis. In von Eye A, Clogg CC, editors. Latent variables analysis: applications for developmental research. Thousand Oaks, CA: Sage.

Smith PM, Oudyk J, Potter G, Mustard C. (2020) The Association between the Perceived Adequacy of Workplace Infection Control Procedures and Personal Protective Equipment with Mental Health Symptoms: A Crosssectional Survey of Canadian Health-care Workers during the COVID-19 Pandemic: L'association entre le caractere adequat percu des procedures de controle des infections au travail et de l'equipement de protection personnel pour les symptomes de sante mentale. Un sondage transversal 
des travailleurs de la sante canadiens durant la pandemie COVID-19. Can J Psychiatry; 706743720961729.

Soklaridis S, Lin E, Lalani Y et al. (2020) Mental health interventions and supports during COVID- 19 and other medical pandemics: a rapid systematic review of the evidence. Gen Hosp Psychiatry; 66: 133-46.

Vaishnavi S, Connor K, Davidson JR. (2007) An abbreviated version of the Connor-Davidson Resilience Scale (CD-RISC), the CD-RISC2: psychometric properties and applications in psychopharmacological trials. Psychiatry Res; 152: 293-7.
Wang C, Pan R, Wan X, Tan Y, Xu L, Ho CS, Ho RC. (2020) Immediate psychological responses and associated factors during the initial stage of the 2019 Coronavirus Disease (COVID-19) epidemic among the general population in China. Int J Environ Res Public Health; 17: 1729.

Wright HM, Griffin BJ, Shoji K et al. (2020) Pandemic-related mental health risk among front line personnel. J Psychiatr Res; 137: 673-80.

Zigmond AS, Snaith RP. (1983) The hospital anxiety and depression scale. Acta Psychiatr Scand; 67: 361-70. 